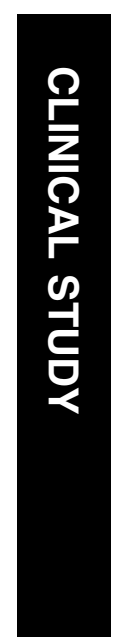

\title{
Psychological distress in people with disfigurement from facial palsy
}

\begin{abstract}
Aims Psychological distress is well documented in people with facial disfigurement. However, the prevalence of psychological distress in patients with facial palsy has not been studied. This study aims to establish the prevalence of psychological distress and the extent of anxiety and depression in a sample of facial palsy patients from the Northwest of England.

Method A total of 103 participants with facial palsy completed a questionnaire pack comprising the Illness Perception Questionnaire-Revised (IPQ-R), a demographic questionnaire, and the Hospital Anxiety and Depression Scale (HADS). The severity of participants' facial palsy was measured by the House-Brackmann scale. Results In all, 32.7 and $31.3 \%$ of the sample had significant levels of anxiety and depression, respectively. The mean age of participants was 59 , and $35.9 \%$ had grade 6 facial palsy. Significant associations were found between participants' perception of consequences, duration, timeline, and the level of distress. No significant associations were found between clinical severity of facial palsy and levels of distress. Females had significantly higher levels of anxiety compared with males.

Conclusions There was a significant level of distress in this study group. The levels of psychological distress were higher than the levels found in other outpatient attenders. There were significant associations between participants' illness perceptions and their level of distress.

Eye (2011) 25, 1322-1326; doi:10.1038/eye.2011.158; published online 1 July 2011
\end{abstract}

Keywords: facial paralysis; psychological distress; facial palsy; facial disfigurement; anxiety; depression
Introduction

Patients with facial disfigurement from post-operative head and neck cancer, ${ }^{1}$ Grave's Ophthalmopathy, ${ }^{2}$ and burns ${ }^{3}$ experience psychological distress (defined as levels of anxiety and depression for this study). However, these studies have demonstrated that there is no direct relationship between the objective degree of disfigurement and the subjective degree of distress experienced..$^{4,5}$

Facial palsy is a form of facial disfigurement and patients can experience severe psychological and social problems. ${ }^{6}$ Their facial expression is either diminished or altered, affecting normal face-to-face communication with others, and non-verbal facial signs can be misinterpreted. ${ }^{7}$ Functional problems such as eating and drinking can cause embarrassment. ${ }^{8}$ The functional significance of the facial palsy extends to the eye region; a functionally dry eye with an inability to blink can result in corneal damage. ${ }^{9}$ It has been noted that it is the inability to smile and express emotion that is most distressing to facial palsy patients. ${ }^{10}$

Evidence in the literature shows that these problems lead to greater levels of anxiety, depression, maladaptive behaviours, and reduced emotional well-being, suggesting that a person's psychological adjustment to the facial disfigurement is a key indicator of overall recovery. ${ }^{11,12}$ Hence, there is a need to measure and model patients' beliefs and thought processes regarding their condition.

Leventhal et al's self-regulatory model is a health-information-processing model based on a person's own common-sense beliefs about their illness. ${ }^{13}$ It is made up of three main constructs: illness representations, coping, and appraisal. The illness representations are organised into five dimensions of illness experience: cause, consequences, cure or control, timeline, and identity. In the context of this study they become (1) perceived causes 
of facial palsy, (2) perceived consequences of facial palsy, (3) beliefs about its cure or control, (4) beliefs about the duration or course of the facial palsy, and (5) symptoms that participants believe are related to their facial palsy. In the model, participants' beliefs about their facial palsy are formed from information obtained from health professionals plus information from family, friends, and the media. These health beliefs are formed early on and may be more or less resistant to change. Patients develop emotional responses to beliefs about their altered facial appearance, which informs coping behaviour. ${ }^{13}$

The extent of psychological distress in patients with facial palsy is not known. Previous studies focused on social problems and patient satisfaction after a particular surgical intervention. ${ }^{14}$ These studies tended to have very small sample groups and the methods used to measure distress were not uniform. ${ }^{14,15}$ In addition, it has been shown that the extent of psychological problems due to patients' facial palsy was underestimated by their clinicians; some patients still experience significant levels of distress after a good surgical result. ${ }^{10}$ This study aims to look at the levels of psychological distress in facial palsy patients, and whether participants' illness representations influence their emotional adjustment.

\section{Materials and methods}

Ethical approval was obtained for this cross-sectional study. Data collection took place over 12 months from facial palsy patients attending a tertiary referral centre in a teaching hospital in the North West of England. Inclusion criteria were as follows: (1) a diagnosis of facial palsy; (2) an adequate level of English to complete the measures; and (3) facial palsy of more than 6 months in duration. Patients who were under 18 years, with a diagnosis of psychiatric illness or currently receiving treatment for a psychological problem, were excluded from the study. The clinical severity of their facial palsy was measured using the House-Brackmann scale. ${ }^{16}$

\section{Measures}

The Illness Perception Questionnaire-Revised (IPQ-R) assesses patients' beliefs about their condition and includes 14 items that assess symptoms (identity). ${ }^{17}$ There are eight subscales: identity, timeline, causes, consequences, personal control, treatment control, illness coherence (patient's understanding of their illness), and emotional representation. The timeline dimension is further divided into an acute/chronic/cyclical subscale. Higher scores for the timeline and consequences subscales indicate a stronger belief that the illness is chronic and has greater consequences on the patient's quality of life. Higher scores on the personal control and treatment control subscales indicate that the patient believes that they have a greater degree of control over their illness and that the treatment is more effective. Illness coherence is a measure of how well the patient understands their illness, with higher scores denoting greater understanding. Finally, emotional representation assesses distress, with higher scores indicating greater distress. The IPQ-R has already been validated in 11 illness groups but has not yet been validated in facial palsy patients. ${ }^{17}$ The Hospital Anxiety and Depression Scale (HADS) consists of a 14-item scale (two seven-item subscales). The total score for each subscale ranges from 0 to $21 .{ }^{18}$ Different cutoff points are used, but it is generally agreed that a score of $\leq 7$ corresponds to a noncase, a score of 8-10 to a probable case, and a score of $\geq 11$ to a definite case. The HADS has been well validated and is commonly used to screen patients with a medical illness. It excludes somatic symptoms of anxiety and depression, which may overlap with the physical illness. $^{18}$

\section{Procedure}

Participants were briefed on the nature of the study, and gave consent to complete the measures, which consisted of a demographic questionnaire, IPQ-R, and HADS. Any subsequent relevant missing information was extracted from the patient notes.

\section{Statistical analysis}

Data were analysed with SPSS v16 (SPSS Inc., Chicago, IL, USA), incorporating descriptive statistics, correlations, and group comparisons. Correlation analysis between the different subscales of the IPQ-R, and between the IPQ-R subscales and HAD subscales were undertaken using Spearman's $\rho$. Correlations were also sought between relevant demographic variables and each of the measure variables.

\section{Results}

\section{Sample characteristics}

A total of 168 facial palsy patients were asked to take part in the study, with 103 completing the questionnaire pack. The participants were aged between 18 and 93 years $(M=59 ; \mathrm{SD}=17)$. The mean age of onset was 54 years. Duration of facial palsy from onset ranged from 6 months to 50 years. There was great variation in the causes of the sample's facial palsy; however, the majority were due to either acoustic neuroma surgery or Bell's Palsy (Figure 1). In all $52 \%$ of the sample's facial palsy was as a 


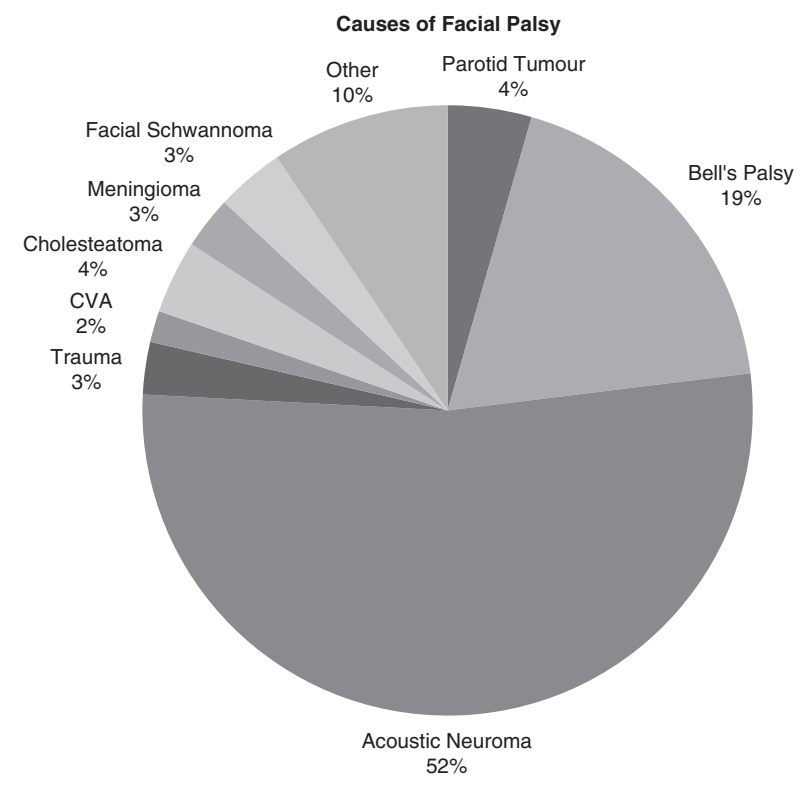

Figure 1 Causes of facial palsy.

Table 1 Level of psychological distress according to HADS score

\begin{tabular}{lcc}
\hline HADS score & HADS anxiety (\%) & HADS depression $(\%)$ \\
\hline$<7$ (non-case) & $66(67.3)$ & $66(68.8)$ \\
$8-10$ (mild) & $17(17.3)$ & $16(16.7)$ \\
$11-15$ (moderate) & $10(10.2)$ & $11(11.5)$ \\
$>16$ (severe) & $5(5.1)$ & $3(3.1)$ \\
\hline
\end{tabular}

result of acoustic neuroma treatment, followed by $19 \%$ caused by Bell's Palsy.

\section{Levels of distress}

Psychological distress was measured using the HAD scale (Table 1). This scale is split into normal, mild, moderate and severe anxiety and depression. For the participants, approximately a third were identified as having a depressive disorder $(31.3 \% ; N=30)$. Of these, $16 \%(N=16)$ had mild depression. The respective confidence interval for anxiety and depression scores was \pm 7.36 per cent. A total of 11 participants were moderately depressed and 3 were severely depressed. In all $32.7 \%(N=32)$ of the participants were identified as having an anxiety disorder, with $17.3 \%(N=17)$ being mild, $11.5 \%(N=11)$ being moderate, and $3.1 \%(N=3)$ being severe.

The clinical severity of facial palsy was assessed using the House-Brackmann scale with grades from 1 to 6 ; 1 being normal and 6 corresponding to complete facial paralysis at the time of surgery. The majority of
Table 2 Clinical severity of facial palsy

\begin{tabular}{lc}
\hline House-Brackmann scale & Frequency (\%) \\
\hline Grade 1 & $2(2.2)$ \\
Grade 2 & $7(7.6)$ \\
Grade 3 & $19(20.7)$ \\
Grade 4 & $14(15.2)$ \\
Grade 5 & $17(18.5)$ \\
Grade 6 & $33(35.9)$ \\
\hline
\end{tabular}

participants had moderate-to-severe facial palsy; $35.9 \%$ of participants had grade 6 facial palsy (Table 2).

\section{Correlation analysis}

There was no correlation found between the clinical severity of facial palsy and participants' experience of anxiety or depressive symptoms. Similarly, there was no correlation between the clinical severity and participants' emotional representations. Significant correlations between the illness perception subscales were found (Table 3). The more that participants believed their illness was chronic, the more they believed the consequences of their facial palsy had an impact on quality of life. Illness chronicity was negatively correlated with both of these beliefs (personal control and treatment control). There was also a significant positive correlation between the timeline (cyclical) and the two control subscales.

There were significant correlations between some of the illness representations and HAD subscales (Table 4). The more the consequences that the participants reported, the more the anxiety and depression symptoms that they experienced. Participants' emotional representation was positively correlated with both HAD subscales, which confirmed that participants' distress regarding their facial palsy measured by the IPQ-R was correlated with distress as measured by the HADS. The duration of participants' facial palsy was significantly correlated to the levels of depression but not to the level of anxiety experienced. The gender of the participants affected their anxiety but not depression scores.

\section{Comparative analysis}

A Mann-Whitney $U$ test was conducted to compare the HADS scores for male and female participants. There was a significant gender difference in HADS scores $(P=0.023, U=770)$; males and females differed significantly in terms of their overall psychological distress. When tested separately, only HADS anxiety scores were significantly different $(P=0.004, U=727)$. Female participants experienced significantly higher levels of anxiety compared with male participants. 
Table 3 Significant correlations between IPQ-R subscales

\begin{tabular}{|c|c|c|c|c|c|c|c|c|}
\hline Spearman's $\rho$ & Duration & $\begin{array}{l}\text { Timeline } \\
\text { cyclical }\end{array}$ & Consequences & $\begin{array}{c}\text { Personal } \\
\text { control }\end{array}$ & $\begin{array}{c}\text { Treatment } \\
\text { control }\end{array}$ & $\begin{array}{l}\text { Illness } \\
\text { coherence }\end{array}$ & $\begin{array}{c}\text { Emotional } \\
\text { representation }\end{array}$ & Timeline \\
\hline Duration & - & - & - & - & - & - & $0.207^{*}$ & $0.482^{* *}$ \\
\hline Timeline cyclical & - & - & - & $0.280^{* *}$ & $0.314^{* *}$ & - & $0.232^{*}$ & - \\
\hline Consequences & - & - & - & - & - & - & $0.578^{* *}$ & $0.300^{* *}$ \\
\hline Personal control & - & $0.280^{* *}$ & - & - & $0.577^{* *}$ & - & - & $-0.245^{*}$ \\
\hline Treatment control & - & $0.314^{* *}$ & - & $0.577^{* *}$ & - & - & - & $-0.203^{*}$ \\
\hline Illness coherence & - & - & - & - & - & - & - & - \\
\hline Emotional rep & $0.207^{*}$ & $0.232^{*}$ & $0.578^{* *}$ & - & - & - & - & $0.441^{* *}$ \\
\hline Timeline & $0.482^{* *}$ & - & $0.300^{* *}$ & $-0.245^{*}$ & $-0.203^{*}$ & - & $0.441^{* *}$ & - \\
\hline
\end{tabular}

${ }^{*} P<0.05$.

${ }^{* *} P<0.01$.

Table 4 Significant correlations between IPQ-R subscales, HAD subscales, and relevant demographic variables

\begin{tabular}{lccc}
\hline Spearman's $\rho$ & $\begin{array}{c}\text { HADS } \\
\text { anxiety }\end{array}$ & $\begin{array}{c}\text { HADS } \\
\text { depression }\end{array}$ & $\begin{array}{c}\text { HADS } \\
\text { (combined) }\end{array}$ \\
\hline HB Scale & - & - & - \\
Duration & - & $0.224^{*}$ & $0.230^{*}$ \\
Gender & $0.292^{* *}$ & - & $0.233^{*}$ \\
Age & - & - & - \\
Marital status & - & - & - \\
Timeline & - & $0.225^{*}$ & $0.232^{*}$ \\
Timeline cyclical & - & $0.285^{* *}$ & $0.253^{*}$ \\
Consequences & $0.359^{* *}$ & $0.403^{* *}$ & $0.436^{* *}$ \\
Personal control & - & - & - \\
Treatment control & - & - & - \\
Ill coherence & - & - & - \\
Emotional rep & $0.581^{* *}$ & $0.594^{* *}$ & $0.631^{* *}$ \\
\hline
\end{tabular}

${ }^{*} P<0.05$.

${ }^{* *} P<0.01$.

There were no significant differences between male and female participants' illness representations. There was no significant difference across the different clinical severity grades in the levels of anxiety and depression as well as illness representations. There was no significant difference between marital status and anxiety, depression and illness representation scores.

\section{Discussion}

This study shows that a significant proportion of participants with facial palsy, experience psychological distress as a result of their condition. The distress levels in this sample were significantly higher than in other HADS populations. ${ }^{18}$ A recent study by Bradbury et $a l^{10}$ found distress levels of $11.4 \%$ for depression and $35 \%$ for anxiety, respectively, in a sample of facial palsy patients. Our participants had higher levels of depression compared with the Bradbury study, but slightly lower levels of anxiety.

The duration of participants' facial palsy was calculated as the time between diagnosis and the date of completion of the questionnaire. There were some significant correlations between the duration and the timeline and emotional representation subscales of the IPQ-R, as well as the HADS. This indicated that the duration of their facial palsy affected participants' levels of distress. We are planning an intervention study to look at possible changes in levels of distress and illness perceptions over time.

Other research shows that females report greater psychological distress, possibly owing to the greater emphasis placed on their appearance by society. ${ }^{19,20}$ In this study there was a significant gender difference in terms of the HADS anxiety scores only. Female participants had significantly higher levels of anxiety compared with male participants. Female and male participants were equally likely to experience high levels of depression. As expected, emotional representation was significantly correlated with the HADS levels of distress, indicating that participants' distress regarding their illness beliefs is consistent across measures, and is not an artefact of one measure.

Obtaining enough numbers for the study was a challenge because facial palsy is not a common condition. Only a small proportion of people have permanent facial palsy as used in this study. Practical and ethical considerations meant that most of the participants were recruited via the healthcare system. Therefore, people who did not actively seek treatment were automatically excluded, because they had mild facial palsy or were not willing to seek treatment, or both. Participants in this study may have more severe facial palsy and be more badly affected owing to the nature of recruitment.

\section{Conclusion}

To conclude, this study has found significant levels of psychological distress in some of our participants. There were widespread low levels of psychological distress in the sample as a whole. Significant correlations were found between some of the participants' perceptions of 
their facial palsy and their level of psychological distress. Significant correlations were also found between some of the demographic variables and participants' level of distress and illness perceptions. There were also significant differences in anxiety scores between female and male participants.

It has become clear that treating the functional problems of facial palsy alone is not enough. Although the functional problem might be resolved, the social and psychological problems will remain. There is growing evidence demonstrating the centrality of the face in communication, identity, and an individual's sense of self. ${ }^{21}$ There is strong evidence that unplanned alteration of the face will cause psychosocial problems. ${ }^{22}$ However, health service provision has not fully understood or embraced this research. ${ }^{23}$ We hope that this study will enable clinicians to better understand their patients, and to manage them according to their overall needs.

\section{Summary}

What was known before

- Psychological distress is documented in people with facial disfigurement.

\section{What this study adds}

- Level of psychological distress in another disease group: facial palsy. Implications for clinical care.

\section{Conflict of interest}

The authors declare no conflict of interest.

\section{Acknowledgements}

This work was supported by the BUPA Foundation (Philip Poole-Wilson Seed Corn Fund) and the NIHR Biomedical Research Centre (Manchester).

\section{References}

1 Callahan C. Facial disfigurement and sense of self in head and neck cancer. Soc Work Health Care 2004; 40(2): 73-87.

2 Farid M, Roch-Levecq AC, Levi L, Brody BL, Granet DB, Kikawa DO. Psychological disturbance in graves ophthalmology. Arch Ophthalmol 2006; 124(3): 426-427.

3 White AC. Psychiatric study of patients with severe burn injuries. BMJ (Clinical Research ed.) 1982; 284(6314): 465-467.

4 Macgregor FC. After Plastic Surgery: Adaptation and Adjustment. Praeger: New York, 1979.

5 Bradbury E. Understanding the Problems. Visibly Different: Coping with Disfigurement. Butterworth-Heinemann: Oxford, 1997, pp 180-193.
6 Cross T, Sheard CE, Garrud P, Nickolopoulos TP, O'Donoghue GM. Impact of facial paralysis on patients with acoustic neuroma. Curr Surg 2005; 62(2): 156-161.

7 Keillor JM, Barrett AM, Crucian GP, Kortenkamp S, Heilman KM. Emotional experience and perception in the absence of facial feedback. Journal of the International Neuropsychological Society: JINS 2002; 8(1): 130-135.

8 Weir A, Pentland B, Crosswaite A, Murray J, Mountain R. Bell's palsy: the effect on self-image, mood state, and social activity. Clin Rehabil 1995; 9(2): 121-125.

9 Rahman I, Sadiq SA. Ophthalmic management of facial nerve palsy: a review. Surv Ophthalmol 2007; 52(2): 121-144.

10 Bradbury ET, Simons W, Sanders R. Psychological and social factors in reconstructive surgery for hemi-facial palsy. J Plastic Reconstr Aesth Surg 2006; 59(3): 272-278.

11 Anderson G. Anxiety, optimism, and symptoms reporting following surgery for acoustic neuroma. J Psychosom Res 1999; 46(3): 257-260.

12 Van Swearingen JM, Cohn J, Turnbull J, Mirzai T, Johnson P. Psychological distress: linking impairment with disability in facial neuromotor disorders. Otolaryngol Head Neck Surg 1998; 118(6): 790-796.

13 Leventhal H, Benyamini Y, Brownlee S, Diefenbach M, Leventhal EA, Patrick-Miller L et al. Illness Representations: Theoretical Foundations. Perceptions of Health and Illness. Harwood Academic Publishers: Australia, 1997, pp 19-46.

14 Kiese-Himmel C, Laskawi R, Wrede S. Psychosocial problems and coping with illness by patients with defective hearing after facial paralysis. HNO 1993; 41(5): 261-267.

15 Hirschenfang M, Goldberg M, Benton J. Psychological aspects of patients with facial paralysis. Dis Nerv Syst 1969; 30: 257-261.

16 House JW, Brackmann DE. Facial nerve grading system. Otolaryngol Head Neck Surg 1985; 93(2): 146-147.

17 Moss-Morris R, Weinman J, Petrie KJ, Horne R, Cameron LD, Buick D. The revised illness perception questionnaire (IPQ-R). Psychol Health 2002; 17(1): 1-16.

18 Zigmond AS, Snaith RP. The hospital anxiety and depression scale. Acta Psychiatr Scand 1983; 67(6): 361-370.

19 Kuehner C. Gender differences in unipolar depression: an update of epidemiological findings and possible explanations. Acta Psychiatr Scand 2003; 108(3): 163-174.

20 Macgregor FC. Social psychological and cultural dimensions of cosmetic and reconstructive plastic surgery. Aesth Plast Surg 1989; 13(1): 1-8.

21 Jackson LA. Psychical attractiveness: a sociocultural perspective. In: Pruzinsky TFCT (ed). Body Image: A Handbook of Theory, Research, and Clinical Practice. Guilford Press: New York, 2002, pp 13-21.

22 Morgan SR, Byrne P. The importance of facial expression and the management of facial nerve injury. Neurosurg $Q$ 2004; 14(4): 239-248.

23 Pritchard C, Clapham L, Davis A, Lang DA, Neil-Dwyer G. Psycho-socio-economic outcomes in acoustic neuroma patients and their carers related to tumour size Cln Otolaryngol 2004; 29: 324-330. 